This is an electronic reprint of the original article. This reprint may differ from the original in pagination and typographic detail.

Author(s): Isoniemi, Tommi; Johansson, Andreas; Toppari, Jussi; Kunttu, Henrik

Title: $\quad$ Collective optical resonances in networks of metallic carbon nanotubes

Year: $\quad 2013$

Version:

Please cite the original version:

Isoniemi, T., Johansson, A., Toppari, J., \& Kunttu, H. (2013). Collective optical resonances in networks of metallic carbon nanotubes. Carbon, 63, 581-585. https://doi.org/10.1016/j.carbon.2013.07.018

All material supplied via JYX is protected by copyright and other intellectual property rights, and duplication or sale of all or part of any of the repository collections is not permitted, except that material may be duplicated by you for your research use or educational purposes in electronic or print form. You must obtain permission for any other use. Electronic or print copies may not be offered, whether for sale or otherwise to anyone who is not an authorised user. 


\title{
Collective optical resonances in networks of metallic carbon nanotubes
}

\author{
T. Isoniemi ${ }^{1}$, A. Johansson ${ }^{1}$, J. J. Toppari ${ }^{1, *}$, and H. Kunttu ${ }^{2}$ \\ Nanoscience Center, ${ }^{1}$ Department of Physics and ${ }^{2}$ Department of Chemistry, \\ P.O.Box 35 (YN), FI-40014 University of Jyväskylü, Finland
}

(Dated: June 17, 2013)

\begin{abstract}
We demonstrate that thin films of randomly oriented metallic single-walled carbon nanotubes possess optical resonances with significant dispersion. The resonances are observed in the Kretschmann configuration as minima in reflection spectra close to $400 \mathrm{~nm}$ and $700 \mathrm{~nm}$ wavelengths. The dispersions are visible only when the material is excited with $s$-polarized light, and most prominent in layers with thickness near $100 \mathrm{~nm}$. We conclude that magnetic plasmon polaritons arising from intertube interactions are a likely explanation. Closeness of the $\mathrm{M}_{11}$ and $\mathrm{M}_{22}$ transition energies to the observed resonances points to a possible coupling with excitons.
\end{abstract}

Carbon nanotubes (CNTs) have gathered huge interest due to their unique mechanical and electric properties. However, they show great promise also for new optical nanostructures. Due to the van Hove singularities in their density of states, they have distinct optical resonances with excitonic characteristics [1]. In addition, CNTs may also possess plasmonic properties. In electron energy loss spectroscopy $\pi$-plasmons have been observed in unsorted single walled (SW) CNTs showing a prominent dispersion between the energies 5 and $10 \mathrm{eV}$; and $\pi+\sigma$-plasmons appearing similarly at $15-25 \mathrm{eV}[2]$.

Despite a variety of theoretical predictions (see [3] and references therein), few experimental results exist on optical plasmons in CNT materials. Surface plasmon polaritons (SPPs) excited by a commonly used total internal reflection (TIR) setup, the Kretschmann configuration (see Fig. 1a), have been shown to couple through an array of vertical multi-walled CNTs [4]. In addition, composite materials of SWCNTs can show localized surface plasmon resonances (LSPRs) dependent on tube length [5].

In this article we demonstrate dispersive and polarization dependent optical modes excited via the Kretschmann configuration within a randomly oriented planar network of metallic SWCNTs.

The used SWCNT materials were purchased from NanoIntegris Inc. (Menlo Park, CA, USA), and consisted of arc discharge produced SWCNTs with a mean diameter of $1.4 \mathrm{~nm}$. The SWCNTs had been enriched to either high metallic or semiconducting concentrations (98\%) using density gradient ultracentrifugation. The material was deposited on glass slides as described in the Supplementary material. The formed layers consist of randomly oriented CNT networks consisting mainly of bundles, as seen in Fig. $1 \mathrm{~b}$ and $\mathrm{c}$.

Reflection spectroscopy was performed on the SWCNT networks, using the Kretschmann configuration with a varying excitation angle and a collimated white light source, as shown in Fig. 1a and Supplementary material. The measurements with $s$-polarized light showed a

\footnotetext{
*Corresponding author. E-mail address: j.jussi.toppari@jyu.fi
}
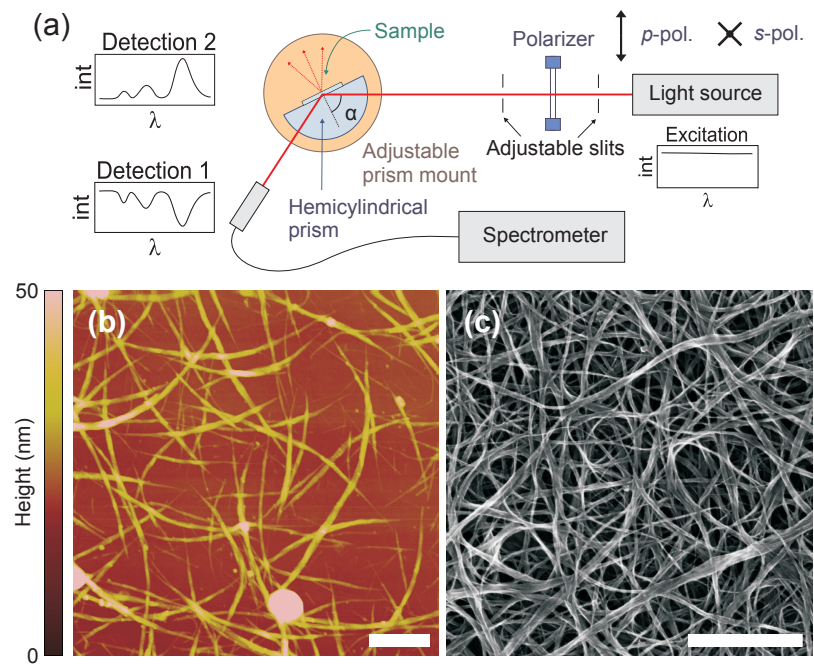

FIG. 1: (a) Schematics of the measurement setup utilizing Kretschmann configuration. (b) Atomic force microscope (AFM) image of a typical thin network of metallic SWCNTs. (c) Scanning electron micrograph of a thick network of metallic SWCNTs. All the scale bars are $500 \mathrm{~nm}$.

clear dip moving from 600 to $800 \mathrm{~nm}$ as the excitation angle, $\alpha$, relative to the sample normal increased (see Figs. 2 and 3a). A similar feature was also found around $400 \mathrm{~nm}$. With $p$-polarized excitation no clear dispersion was observed, and only a spectrum similar to the transmission of the sample was visible as shown in Fig. 3a. Control samples prepared similarly but using semiconducting (Fig. 3d) and unsorted (Supplementary material) SWCNTs did not display any dispersive features.

To test the influence of the surrounding medium on the resonances, the metallic SWCNT layers were measured within additional media. When embedding the SWCNT film in isopropanol $\left(n_{\text {air }}=1.00 \rightarrow n_{\text {IPA }}=1.38\right)$, the dispersive resonances appeared at the same energies, but with shifted and more prominent angle dependence, as shown in Figs. 3b and 2b. Changing the surrounding medium to water yielded similar response (see Supplementary material). Instead, evaporation of $50 \mathrm{~nm}$ of gold on top of the SWCNT film destroyed any previous dispersive resonances: only the stagnant transmission spectrum 

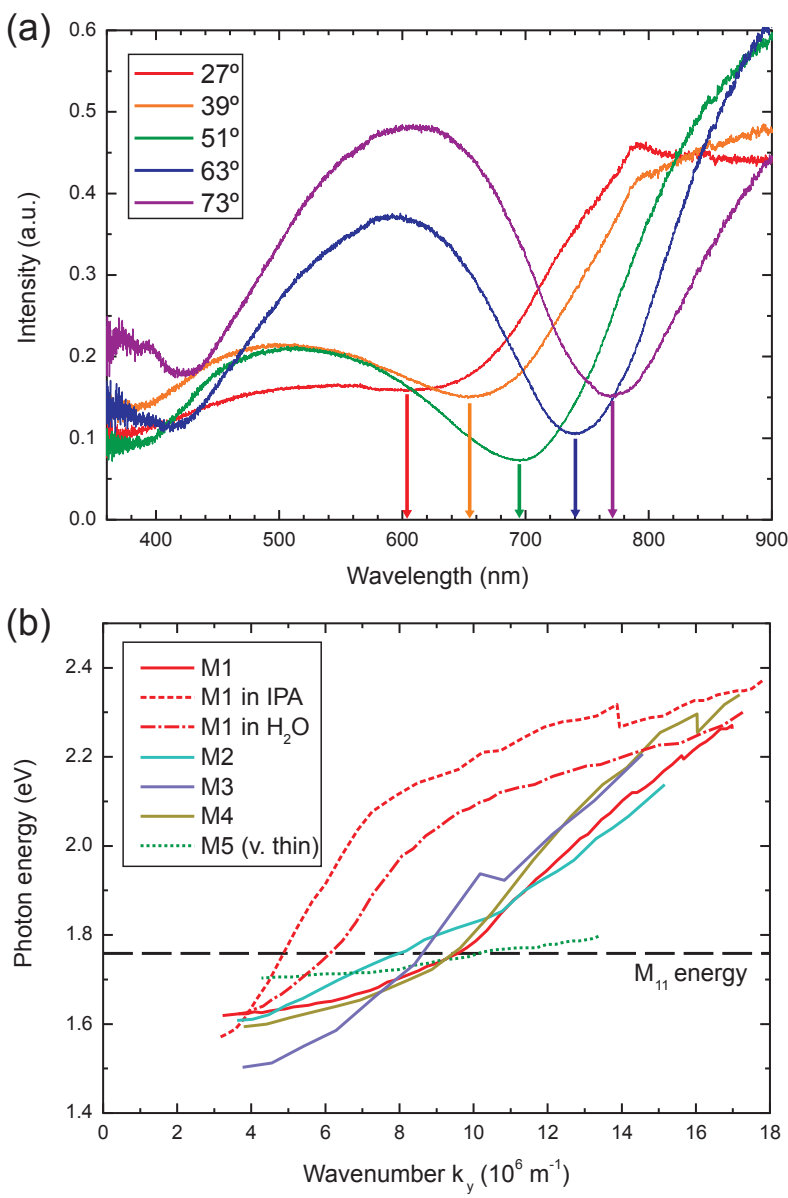

FIG. 2: (a) Reflection spectra from a metallic SWCNT network (sample M2). Excitation angles are given relative to the sample normal. (b) Dispersion curves, i.e., minimum of the dip in the reflectance spectrum as a function of a vertical component of the wavevector, $k_{y}$, measured from various metallic SWCNT film samples. M1 is measured in air, as well as embedded in isopropanol and water; others only in air. Samples M1-M4 are roughly $100 \mathrm{~nm}$ thick, while M5 is only $5 \mathrm{~nm}$ thick.

of the SWCNTs was visible at $s$-polarization, and the characteristic surface plasmon resonance of a gold layer is seen with $p$-polarized excitation, as shown in Fig. 3c. The described resonances have also a clear dependence on the thickness of the SWCNT layer, being more pronounced near $100 \mathrm{~nm}$ thickness, see Fig. 4. Very thin ( $\leq$ $20 \mathrm{~nm})$ and thick $(\geq 0.5 \mu \mathrm{m})$ layers do not have the dispersive resonances in their reflectivity spectra as shown in Fig. 2b, where the observed dispersions of different samples and environments are collected.

In our case the resonances are observed at $s$ polarization, where the electric field of the optical excitation is parallel to the rotation axis of the Kretschmann configuration, and changing the angle does not affect its projection. On the other hand, the magnetic field of an $s$-polarized photon is perpendicular to the CNT film, and thus the angle makes a difference in terms of momentum,
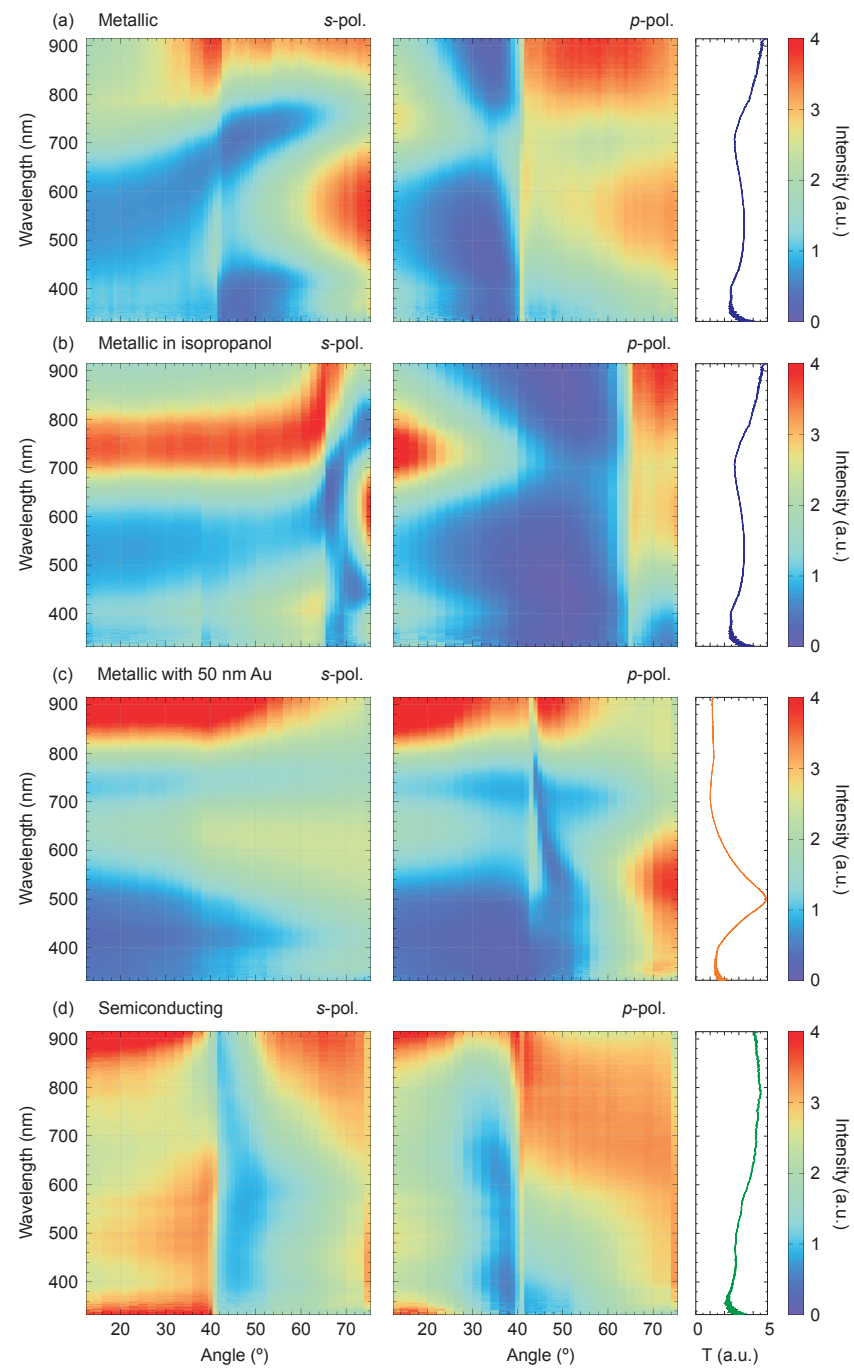

FIG. 3: Intensity of reflected light as a function of the excitation angle and the wavelength for $\sim 100 \mathrm{~nm}$ thick SWCNT networks. (a) Sample M1, which has metallic SWCNTs. The observed resonances are visible with $s$-polarization at angles above the TIR angle, $\sim 41^{\circ}$. (b) M1 embedded in isopropanol. The resonances appear again with $s$-polarization at angles above the TIR angle, $\sim 65^{\circ}$. (c) Sample M4 with metallic SWCNTs and a $50 \mathrm{~nm} \mathrm{Au}$ coating on top. Typical SPP resonance is visible at $p$-polarization. (d) Sample with semiconducting SWCNTs. Transmission spectrum for each sample is also visible on the right, except for (b), where transmission was measured without isopropanol.

and could imply a dispersive behavior. One possible interpretation agreeing with the observed phenomenon is thus magnetic resonances within the network [6], excited by the magnetic field of the excitation light.

The thickness dependence of the resonances further points to a collective resonance similar to electric SPPs, which can only be excited with $p$-polarized light in thin metal films. If the metal is too thin in the case of SPPs excited via the Kretschmann configuration, the SPP will be weak because of radiation damping into the glass. As 

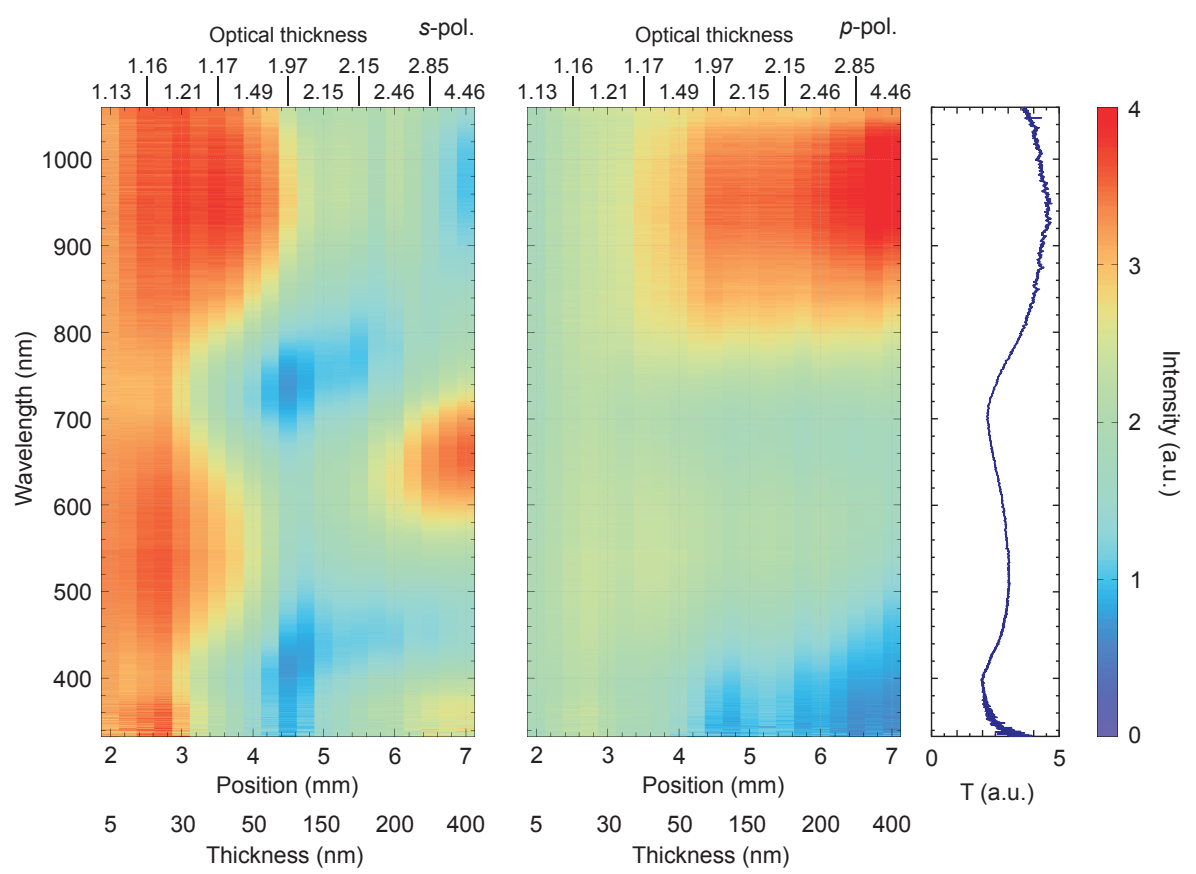

FIG. 4: Intensity of the reflected light as a function of the network thickness and wavelength for metallic SWCNTs. Excitation angle is $51^{\circ}$. Optical thickness refers to transmission intensity at $900 \mathrm{~nm}$ wavelength divided by the intensity at $700 \mathrm{~nm}$ (close to the $\mathrm{M}_{11}$ resonance). Position refers to location on the glass sample parallel to the plane of incidence and the thickness is measured with AFM. The transmission spectrum at right is taken at the position $4.5 \mathrm{~mm}$.

for a thick film, the SPP cannot be efficiently excited due to absorption in the metal. Similar mechanics with magnetic fields could produce the same dependence in a SWCNT film if the excitations appear at the surface [7]. These resonances could be for example magnetic plasmon resonances (MPR) formed due to coupling and regular spacing of SWCNTs within bundles. In this case the bundle acts like a lattice of nanowires with uneven endpoints [6]. Further, these resonances could enable a collective magnetization wave to propagate at the surface of the film. These magnetic plasmon polaritons (MPPs) are a likely explanation because $s$-polarized MPPs have strong dispersion whereas $p$-polarized ones are non-dispersive [8]. This interpretation is further supported, in addition to the thickness dependence, by the sensitivity to the dielectric environment, and it would also hint at a negative magnetic permeability in the material [7]. The effective dispersion also changes, because for very thin films at the $\mathrm{M}_{11}$ and $\mathrm{M}_{22}$ transition energies only normal absorption will be visible, which has no angle dependence.

Gap plasmon modes could also explain the observed phenomenon. However, these modes should depend on the length of the gap, i.e., in this case the length of the SWCNTs in bundles. To test this, a new sample was fabricated out of CNTs shortened by an extensive sonication. This resulted in a similar bundled film, but the resonances were still at the same energies; only weakened in intensity, most probably due to the additional amorphous carbon. Furthermore, LSPR in catalyst metal nanoparticles left over from the CNT synthesis would not dis- play dispersion, and any possible combination with CNTs would have resonances dependent on the tube length. Thus, this possibility can also be ruled out.

Notable is that the dispersive resonances around 600$800 \mathrm{~nm}$ and $400 \mathrm{~nm}$ are close to the $\mathrm{M}_{11}$ and $\mathrm{M}_{22}$ transition energies of metallic SWCNTs, respectively (Figs. 2b and $3 \mathrm{a})$. This raises the question whether exciton creation is causing the optical resonances. Excitons can have momentum dependence in periodic systems, but in the measured SWCNT films also the thickness affects the intensity of the resonance, which is not fully consistent with an excitonic resonance. However, an excitonplasmon state could be responsible for the resonances in our system [9]. Graphene sandwiched between glass and polymer has also shown to pose an excess broadband absorption at $s$-polarization in TIR [10]. However, further experimental and theoretical studies are needed to confirm the underlying mechanism of the observed dispersive optical resonance.

In conclusion, we have observed a clearly dispersive optical resonance in reflection spectroscopy of metallic SWCNT films. It appears only for an $s$-polarized excitation source, and is strongest at film thicknesses around $100 \mathrm{~nm}$. The dependence of intensity and dispersion of the resonance on the thickness and the surrounding environment is consistent with creation of magnetic plasmons or MPPs, and the vicinity of $\mathrm{M}_{11}$ and $\mathrm{M}_{22}$ transitions suggests that excitons may be involved in the process.

This work was supported by the Academy of Finland (Project Nos. 135193 and 218182). We thank Jaakko 
Koivisto for acquiring Raman spectra. T.I. thanks the

Finnish National Doctoral Programme in Nanoscience.

[1] Wang F, Dukovic G, Brus LE, Heinz TF. The optical resonances in carbon nanotubes arise from excitons. Science 2005;308(5723):838-41.

[2] Kramberger C, Hambach R, Giorgetti C, Rümmeli MH, Knupfer M, Fink J, et al. Linear plasmon dispersion in single-wall carbon nanotubes and the collective excitation spectrum of graphene. Phys Rev Lett 2008;100(19):196803-6.

[3] Perez R, Que W. Plasmons in isolated single-walled carbon nanotubes. J Phys: Condens Matter 2006;18:3197216

[4] Lu Q, Rao R, Sadanadan B, Que W, Rao AM, Ke PC Coupling of photon energy via a multiwalled carbon nanotube array. Appl Phys Lett 2005;87:173102-4.

[5] Shuba MV, Paddubskaya AG, Plyushch AO, Kuzhir PP, Slepyan GY, Maksimenko SA, et al. Experimental evidence of localized plasmon resonance in composite materials containing single-wall carbon nanotubes. Phys Rev
B 2012;85(16):165435-40.

[6] Sarychev AK, Shvets G, Shalaev VM. Magnetic plasmon resonance. Phys Rev E 2006:73(3):036609-18.

[7] Ruppin R. Surface polaritons of a left-handed medium. Phys Lett A 2000;277(1):61-4.

[8] Li T, Wang SM, Liu H, Li JQ, Wang FM, Zhu SN, et al. Dispersion of magnetic plasmon polaritons in perforated trilayer metamaterials. J Appl Phys 2008;103(2):0231047.

[9] Grüning M, Marini A, Gonze X. Exciton-plasmon states in nanoscale materials: breakdown of the Tamm-Dancoff approximation. Nano Lett 2009;9(8):2820-4.

[10] Ye Q, Wang J, Liu Z, Deng ZC, Kong XT, Xing F, et al. Polarization-dependent optical absorption of graphene under total internal reflection. Appl Phys Lett 2013;102(2):021912-5. 